\title{
David Cohen (1922-2013)
}

Jérôme Lentin et Antoine Lonnet

\section{(2) OpenEdition}

\section{Journals}

Édition électronique

URL : http://journals.openedition.org/beo/1252

DOI : 10.4000/beo.1252

ISSN : 2077-4079

\section{Éditeur}

Presses de l'Institut français du Proche-Orient

\section{Édition imprimée}

Date de publication : 30 avril 2014

Pagination : 9-16

ISBN : 978-2-35159-403-2

ISSN : 0253-1623

\section{Référence électronique}

Jérôme Lentin et Antoine Lonnet, « David Cohen (1922-2013) », Bulletin d'études orientales [En ligne], LXII | 2014, mis en ligne le 11 juin 2014, consulté le 01 mai 2019. URL : http://journals.openedition.org/ beo/1252; DOl : 10.4000/beo.1252 


\section{DAVID COHEN}

\section{(1922-2013)}

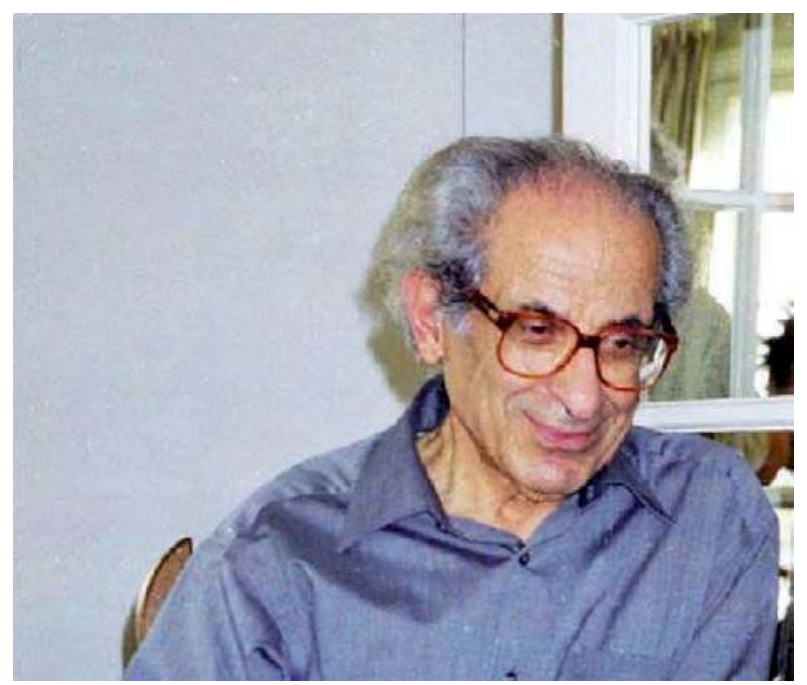

par Jérôme LENTIN (professeur à l'INALCO)

et Antoine LONNET (chargé de recherche au CNRS, UMR 8176)

Évoquer David Cohen, disparu le 9 mars 2013, c'est esquisser le portrait d'un grand savant et d'un homme remarquable.

Tout en s'inscrivant, avec les meilleurs d'une riche génération de linguistes, dans la continuité d'une époque inaugurée par Saussure et Meillet, alliant le structuralisme à la sociologie, il créa, dans le même temps, des orientations d'avenir, par exemple dans l'analyse automatique ou la dialectologie de l'arabe. Grand maître des études historiques et comparatives des langues sémitiques et chamito-sémitiques il fut aussi, et avant tout, un linguiste généraliste (ou un « langagiste », comme il aimait à dire). Dans ses domaines de spécialité, en effet, ses nombreux travaux ont toujours été sous-tendus par une théorie de "l'exercice du langage et des langues », qui s'est perfectionnée à travers eux, mais qu'il ne s'est autorisé à exposer que dans ses toutes dernières publications (et dans de nombreux inédits à paraître). On en retiendra une notion essentielle : «les nécessités de la communication ", où nécessités renvoie aux conditions imposées au langage car 
nécessaires à l'établissement et au fonctionnement de la communication, en même temps qu'aux contraintes matérielles, anatomiques, physiologiques, sociales, etc. qui pèsent sur l'homme et sur les communautés humaines. Ces nécessités expliquent mieux la grammaire que toute hypothèse mécaniste sur les capacités de l'esprit. Le champ où cette notion directrice révèle le plus vivement sa capacité d'approfondissement est la morphogenèse, par laquelle s'engendrent et s'enchaînent les niveaux de la stylistique, de la syntaxe, de la morphologie et du lexique. On trouvera une introduction claire et vivante à sa pensée dans un livre d'entretiens publié en 2010, et de premiers longs développements dans le premier volume (seul paru à ce jour) de ses Essais sur l'exercice du langage et des langues. ${ }^{1}$

La carrière universitaire de David Cohen le porte, après une période au CNRS, à une direction d'études (hébreu, araméen et sémitique comparé) de la IV e section de l'École Pratique des Hautes Études ainsi qu'à la chaire de linguistique sémitique créée pour lui par l'université de la Sorbonne nouvelle (Paris III), à l'initiative de ses collègues, en particulier Gilbert Lazard.

Cette trajectoire, apparemment sans obstacle, était pourtant fort peu probable. David Cohen, né à Tunis en 1922, dans une famille très modeste, connut parfois la misère dans son enfance. Sa brillante intelligence, sa curiosité intellectuelle et sa déjà vaste culture, souvent acquise à l'étal des libraires ou au sacrifice des trois sous du tram, lui permirent, contre toute fatalité sociale et grâce à l'aide de maîtres clairvoyants, de faire des études secondaires. Le statut des Juifs l'empêcha d'aller plus loin; il connut l'internement dans un camp de travail obligatoire de la Tunisie occupée. À la libération de Tunis, en 1943, il put partir pour Alger et commencer une nouvelle vie, où l'engagement politique eut une place prépondérante, essentiellement dans une activité de journaliste pour la presse communiste, et aussi de traducteur et de directeur de collection (chez l'éditeur Edmond Charlot). Il y avait là une effervescence littéraire et intellectuelle exceptionnelle qui enrichit considérablement sa personnalité culturelle. Ses amis s'appelaient Kateb Yacine, Jean Amrouche, Henri Alleg...

Le jeune journaliste, marié et déjà père, traverse pour la première fois la Méditerranée et devient correspondant de presse à Paris, puis libraire. Sa vie s'oriente enfin de façon définitive lorsqu'il rencontre, par les hasards du voisinage et de la politique, le grand linguiste Marcel Cohen, qui ne manque pas de reconnaître en lui celui qui deviendra très vite le collaborateur, le disciple et l'ami proche, le collègue, le successeur. Grâce à lui, David Cohen entre en 1955 au CNRS. Il n'est pas pour autant à l'abri des soucis que lui causent sa nationalité et l'incertitude qu'elle implique pour le sort de sa famille : il ne deviendra français que très tardivement, et au terme d'épreuves qui auront affecté profondément cet homme angoissé.

Entre désarroi et désillusion, mais sans renier ses idéaux, le militant s'efface devant le savant. Ce sont alors des années de travail forcené. Il suit les enseignements les plus variés (ceux de Marcel Cohen bien sûr, de Michel Lejeune, d'Émile Benveniste, d'André DupontSommer...), se liant à beaucoup de ces grands maîtres. Il participe aux travaux du Groupe

1 Les références complètes des ouvrages et articles cités sont données à la fin de cette notice. 
de linguistique marxiste, animé par Marcel Cohen, aux côtés d'Antoine Culioli, Jean Dubois, Michel Gauthier, André-Georges Haudricourt, Gilbert Lazard, Henri Mitterand, Maxime Rodinson, Guy Serbat... Il publie très rapidement ses premiers travaux linguistiques, dont la densité et la maturité étonnent, et lui attirent immédiatement la considération et l'estime de ses pairs, en France comme dans le monde. Dès 1955 il met en chantier son monumental Dictionnaire des racines sémitiques ou attestées dans les langues sémitiques (10 volumes parus), qu'il laisse inachevé (mais dont la parution sera poursuivie, sur la base de son fichier, de ses notes et de ses ébauches, selon son désir). Dès 1963 et 1964 paraissent deux monographies : la première étudie, pour la première fois, le dialecte arabe hassānīya de Mauritanie ; la seconde, entreprise à l'instigation de son maître Marcel Cohen, étudie, pour la première fois également, Le parler arabe des Juifs de Tunis (sa langue maternelle, au sens propre du terme), d'abord par la publication de Textes et documents linguistiques et ethnographiques (le deuxième volume, l'Étude linguistique proprement dite, bien que déjà rédigé à ce moment, ne sera publié, après diverses péripéties, qu'en 1975). Ces travaux, vite devenus des classiques, sont encore aujourd'hui des modèles difficiles à égaler. En 1963 aussi commencent, d'abord à l'appel et sous la houlette du grand arabisant Régis Blachère, autre rencontre déterminante (cet homme réservé ne lui ménagera ni son affection ni son aide), les quarante années d'enseignement à l'École Pratique des Hautes Études, matrice des grands travaux à venir, dont sa thèse de doctorat d'État, La phrase nominale et l'évolution du système verbal en sémitique. Études de syntaxe historique (parue en 1984). Ce titre indique bien la portée de linguistique générale d'une étude unanimement reconnue comme fondatrice. De même L'Aspect verbal (1989), plus général et plus théorique, mais enrichi d'études monographiques fouillées (entre autres sur les dialectes arabes). C'est évidemment à David Cohen que le CNRS confie la responsabilité de diriger le volume des Langues dans le monde ancien et moderne (collection dirigée par Jean Perrot) consacré aux langues chamito-sémitiques, dont il rédige personnellement les deux tiers.

Il importait de dire en commençant que l'œuvre considérable de David Cohen - toute entière écrite dans une langue claire et dense - embrasse de vastes horizons. À l'intention des lecteurs du Bulletin d'Études Orientales, l'accent sera cependant mis dans les lignes qui suivent sur son œuvre d'arabisant. Outre les deux monographies qui ont été évoquées, et l'activité d'enseignement dont il sera question plus loin, celle-ci s'est matérialisée, trop discrètement sans doute, dans les rapports sur ses conférences de l'École Pratique des Hautes Études et dans de très nombreux comptes rendus d'ouvrages (souvent de véritables articles) et, de façon heureusement plus visible, dans une bonne quinzaine d'articles, qu'il faudrait tous citer, tant ils constituent autant de chefs d'œuvre, devenus eux aussi des classiques. « Ad ddād et ambiguïté linguistique en arabe » (1961) demeure la meilleure mise au point et la meilleure analyse d'un problème qui a fait couler beaucoup d'encre, et pas toujours la meilleure; la même année, «Essai d'une analyse automatique de l'arabe », réalisé avec l'aide de ses amis de l'Institut Blaise Pascal du CNRS tout juste créé, est, sauf erreur, le tout premier travail jamais publié sur des questions promises - mais rien n'était moins sûr alors - à un bel avenir (David Cohen fut d'ailleurs membre fondateur 
de l'A.T.A.LA.: Association pour le traitement automatique des langues, où se retrouvaient, parmi les linguistes, Georges Gougenheim, Marcel Cohen, Jean Fourquet, André Martinet, Antoine Culioli, Bernard Pottier, Alexis Rygaloff, Georges Mounin, René L'Hermitte). En 1962, «Koinè, langues communes et dialectes arabes» résout magistralement les nombreux problèmes suscités par le célèbre article publié en 1959 dans Language par Charles A. Ferguson sur « The Arabic koine ». En 1965, la publication d'une communication de 1960 sur «Le système des voyelles brèves dans les dialectes maghribins » dresse un tableau lumineux et synthétique qui rend compte, avec une grande économie de moyens, d'un ensemble de situations diverses et complexes. "Le système phonologique du maltais. Aspects synchroniques et diachroniques » (1966) expose avec clarté, pour la première fois, l'histoire de la phonologie du maltais. David Cohen a consacré plusieurs études à ce dialecte de l'arabe (et à sa littérature), poussant aussi ses élèves à l'étudier. C'est à Malte que se tint, en 1972, le Premier Congrès d'études des cultures méditerranéennes d'influence arabo-berbère, qu'il contribua, avec quelques autres, à organiser. Trop méconnu, son article «Sur le statut phonologique de l'emphase en arabe» (1969) propose, entre autres richesses, une interprétation des termes 'phonétiques' utilisés par les grammairiens arabes médiévaux. "Les formes du prédicat en arabe et la théorie de la phrase chez les anciens grammairiens », publié en 1970 dans le volume des Mélanges offerts à son maître Marcel Cohen et édités par ses soins, est lui aussi méconnu, ou trop souvent mal compris. En 1973 paraît l'article fondateur de la sociolinguistique de l'arabe, «Variantes, variétés dialectales et contacts linguistiques en domaine arabe ». David Cohen, qui avait activement participé à la préparation des «Matériaux pour une sociologie du langage » de Marcel Cohen, a donné des cours mémorables de cette discipline à l'Université de Paris III. Dans le même domaine, ses « Remarques historiques et sociolinguistiques sur les parlers arabes des Juifs maghrébins » apportent une clarification salutaire sur la question générale des dialectes « communautaires ", ainsi que sur des questions particulières comme le supposé « zézaiement » dans les parlers concernés. David Cohen s'est intéressé à tous les dialectes de l'arabe, centraux comme « périphériques ». Parmi ces derniers, il s'est particulièrement intéressé à l'arabe andalou et à Pedro de Alcalá dont il a préparé une traduction de la grammaire (à paraître; voir déjà par exemple «Les structures accentuelles de l'arabe andalou d'après Pedro de Alcalá », avec Arlette Roth).

David Cohen a dirigé de nombreuses thèses sur les dialectes arabes les plus divers. À ce propos, il est important de signaler avec quelle réussite il a su convaincre de nombreux étudiants arabophones qu'un dialecte arabe est un objet d'étude scientifique légitime, face à la langue classique, comme, symétriquement, l'est le texte coranique, ou tout autre texte sacré. Il en a engagé beaucoup à étudier leurs parlers. Dans des contextes souvent peu favorables, ils ont ainsi trouvé le courage de persévérer ; plus d'un(e) est aujourd'hui professeur d'université dans son pays. La dialectologie et la sociolinguistique de l'arabe, que David Cohen ne séparait à vrai dire pas vraiment, et qu'il a véritablement (re)fondées, sont peut-être les domaines dans lesquels il a le plus encouragé ses élèves à s'engager, soutenant les travaux déjà entrepris, appelant de ses vœux, dès le congrès de 
Malte en 1972, la constitution d'un « atlas linguistique et sociolinguistique de l'arabe », mettant au point un « questionnaire différentiel pour la dialectologie arabe », largement diffusé (mais qui ne sera publié qu'en 2000), viatique de toutes celles et tous ceux qu'il s'efforce d'envoyer en mission sur le terrain, encourageant les uns et les autres à dresser des tableaux sociolinguistiques des grandes métropoles arabes, constituant une équipe (CNRS/Paris III) de tous jeunes chercheurs, souvent encore étudiants (ils sont tous devenus aujourd'hui des chercheurs confirmés et s'efforcent de prolonger son œuvre), fondant une revue (Matériaux Arabes et Sudarabiques) pour qu'ils puissent faire connaître leurs premiers travaux, recueillant des fonds documentaires anciens inédits pour qu'ils les exploitent et les publient. David Cohen peut ainsi être assurément considéré comme l'inspirateur de l'AIDA (l'Association Internationale de Dialectologie Arabe), fondée en janvier 1993 lors d'un premier colloque international organisé à Paris à l'initiative de deux de ses élèves, et qui vient (en novembre 2013) de tenir son $10^{\mathrm{e}}$ colloque.

David Cohen était, on l'a dit, un sémitisant, un chamito-sémitisant et un linguiste généraliste. C'est dire que, dans le domaine de la dialectologie arabe, comme dans les autres, ses travaux se situaient toujours dans ces cadres généraux (le titre d'un de ses derniers articles - car il a travaillé et publié jusqu'à la fin - indique bien ces préoccupations : « Du chamito-sémitique aux dialectes arabes - Les systèmes vocaliques et les contraintes syllabiques »). Il souhaitait que ses élèves - à qui il prêtait généreusement des connaissances qui n'étaient pas toujours les leurs - s'inscrivent, dans la mesure du possible, dans les mêmes perspectives. Plusieurs d'entre eux sont d'ailleurs devenus aussi spécialistes de telle(s) ou telle(s) langue(s) sémitique(s) ou chamito-sémitique(s). Et ils l'ont vu œuvrer, malheureusement sans grand succès, pour que se forment par exemple des groupes internationaux de recherche sur l'arabe occidental (andalou, maghrébin, sicilien, maltais) ou, dans un autre domaine, sur les langues (sémitiques et couchitiques) de la Mer Rouge, aire qu'il considérait comme cruciale à plusieurs égards. Parmi les questions générales qui ont retenu à plusieurs reprises son attention, on signalera - car cet aspect de son œuvre a trop souvent été mal lu et donc mal interprété par les arabisants - celle des racines (par exemple dans le fascicule 6 du Dictionnaire des racines sémitiques).

L'évocation rapide faite plus haut de quelques ouvrages majeurs de linguistique chamito-sémitique et de linguistique générale, et ces quelques rappels sur son apport aux études arabes, ne doivent pas faire oublier que David Cohen a marqué d'autres domaines encore de ses contributions, comme celui de la pathologie du langage, qui lui tenait particulièrement à cœur et qu'il a longuement étudiée (avec des études cliniques) dans le cadre d'une collaboration, à l'hôpital Sainte-Anne, avec des neurologues et avec son ami linguiste Michel Gauthier, lui consacrant des articles novateurs (repris dans le volume d'Essais cité), ou encore celui de la poétique, autre domaine cher au cœur de cet homme d'une culture littéraire et artistique immense.

David Cohen laisse une œuvre considérable : une dizaine d'ouvrages (auxquels se sont ajoutés, dans la dernière période, un livre de souvenirs, dont il avait emprunté le beau titre à un poème de Paul Eluard: L'homme Ses bizarres idées de bonheur et, en collaboration, 
une traduction de poésie italienne, seul témoignage publié d'une activité qu'il pratiquait assidûment), plus de 80 articles, les importants rapports de ses conférences à l'École Pratique des Hautes Études, 250 comptes rendus (la plupart donnés à L'Année Sociologique et au Bulletin de la Société de Linguistique de Paris), près de 300 notices bibliographiques (principalement dans Abstracta Islamica entre 1964 et 1975)...

Ce maître était aussi - et c'est là une part constitutive de son œuvre - un incomparable enseignant. Il a ainsi, comme naturellement et sans qu'il l'ait cherché, créé autour de lui un groupe, toujours ouvert, de disciples dont la plupart ne seraient jamais devenus sans lui des chercheurs. Que ce soit dans des cours d'initiation pour les étudiants de première année ou dans les conférences devant le public en partie constitué de collègues à l'École Pratique des Hautes Études, il était enthousiaste, enthousiasmant, convaincant sans recourir jamais aux outils malhonnêtes des bateleurs, attentif à chaque auditeur, jamais las de répondre aux questions, même les plus invraisemblables, et amenant son public à découvrir en même temps que lui, et comme par lui-même, ce qu'il cherchait à faire connaître. Il savait aussi rappeler la juste mesure des choses, apaisant ainsi, par la vertu de la raison et de la bienveillance, les conflits, tels ceux qui ne manquaient pas de se manifester dans des départements universitaires affectés par les événements du Proche-Orient.

Ces qualités lui venaient non seulement d'un goût profond pour la discussion et le partage, mais aussi du fait que rien ne lui était plus étranger et haïssable qu'une hiérarchie instituée entre les êtres. Les arabisants qui ont fait leurs études à Paris et qui ont pu suivre ses cours à l'Université de la Sorbonne Nouvelle Paris III ou à l'École Pratique des Hautes Études, ceux qui ont eu la chance de faire partie du laboratoire du CNRS qu'il a fondé et dirigé s'en souviennent, comme tous ceux qu'il a encouragés, et souvent révélés à euxmêmes, ou encore celles et ceux qui l'avaient assisté dans les tâches administratives (il ne s'y dérobait jamais), et qui lui vouaient une affection indéfectible. Il accordait a priori confiance, estime et respect à ses interlocuteurs. C'est pourquoi, par exemple, il n'a jamais imposé un sujet de thèse ou de mémoire. Ces dispositions se doublaient d'une pratique d'hospitalité généreuse, au point que, pour bien des collègues étrangers, visiter la France c'était aussi rendre visite à David Cohen, à Viroflay, plus tard à Paris puis à Marseille.

C'est dans cette ville, qui lui rappelait, ainsi qu'à son épouse Christiane Buret-Cohen, l'Afrique du Nord de leur enfance, que s'est éteint David Cohen, dans sa quatre-vingtonzième année. Sa disparition laisse un grand vide : il était le seul en France à maîtriser totalement le champ immense des langues chamito-sémitiques (sémitique, égyptien, berbère, couchitique-omotique, tchadique), qu'on appelle aussi afro-asiatiques, et à bénéficier d'une audience et d'un respect internationaux considérables. À ses disciples, devenus souvent ses amis, il laisse ouvertes, au-delà du chagrin, d'innombrables directions de recherche, dans un paysage où les signes de sa présence ne s'effaceront pas. 
David Cohen a reçu en 1986 le prix Volney de l'Académie des Inscriptions et Belles-Lettres pour son livre La phrase nominale..., en 1988, de la même institution, le prix Antoine Meillet pour l'ensemble de son œuvre, et en 2007, la prestigieuse médaille Lidzbarski de la Deutsche Morgenländische Gesellschaft.

\section{Références des ouvrages et articles cités (dans l'ordre de leur apparition ci-dessus)}

Le langage, les langues et les nécessités de la communication. Conversations à Censier - Avec la participation d'Arlette Roth et d'Antoine Lonnet, Éditions Lambert-Lucas, Limoges, 2010, 363 p. [Avec Aziza Boucherit]

Essais sur l'exercice du langage et des langues, volume 1, Communication et langage, Paris, Maisonneuve \& Larose, 2006, XVI+510 p.

Dictionnaire des racines sémitiques ou attestées dans les langues sémitiques. Comprenant un fichier comparatif de Jean Cantineau, 1970-2012, Paris - La Haye, Mouton (fasc. 1 et 2) ; Leuven, Peeters (fasc. 3-10). [avec la collaboration de François BRON et Antoine LONNET à partir du fasc. 3, auxquels s'ajoute Jérôme LENTIN pour le fasc. 10], lettres 'à K, 1298 p.

Le dialecte arabe hassāniya de Mauritanie (parler de la Gabla), avec la collaboration de Mohammed EL CHENNAFI, Paris, Librairie Klincksieck (Études arabes et islamiques. Études et documents V), 1963, IX+293 p.

Le parler arabe des Juifs de Tunis. Textes et documents linguistiques et ethnographiques, Paris - La Haye, Mouton (EPHE - Sorbonne, 6e section, Études juives VII), 1964, X+177 p.

Le parler arabe des Juifs de Tunis. Tome II. Étude linguistique, The Hague-Paris, Mouton (Janua Linguarum, Series Practica 161), 1975, 318 p.

La phrase nominale et l'évolution du système verbal en sémitique. Études de syntaxe historique, Paris, dépositaire Éditions Peeters, Leuven - Paris (Collection linguistique publiée par la Société de Linguistique de Paris LXXIII). 1984, VII+629 p. [réimpr. 2003]

L’Aspect verbal, Paris, Presses Universitaires de France (collection Le linguiste), 1989, x+272 p.

Les langues chamito-sémitiques (Troisième partie de Les langues dans le monde ancien et moderne, ouvrage publié sous la direction de Jean PERROT), textes réunis par David COHEN, Paris, Éditions du CNRS, 1988, [VIII] +318 p. in $-4^{\circ}, 3$ cartes +2 cartes dépliantes.

"Aḍdād et ambiguïté linguistique en arabe », Arabica 8/1, 1961, p. 1-29.*

"Essai d'une analyse automatique de l'arabe », La traduction automatique 2, 1961, p. 48-70.*

«Koinè, langues communes et dialectes arabes », Arabica 9/2, 1962, p. 119-144.*

«Le système des voyelles brèves dans les dialectes maghribins », Communications et Rapports du Premier Congrès international de dialectologie générale III, Louvain - Bruxelles, août 1960, organisé par Sever Pop, publ. par A. J. van Windekens, Centre international de dialectologie générale, 1965, p. 7-14.*

"Le système phonologique du maltais. Aspects synchroniques et diachroniques ", Journal of Maltese Studies 3, 1966, p. 1-26.*

«Sur le statut phonologique de l'emphase en arabe », Linguistic Studies presented to André Martinet III. NonIndo-European Linguistics (= Word 25/1-3), éd. A. Juilland, 1969, p. 59-69.

«Les formes du prédicat en arabe et la théorie de la phrase chez les anciens grammairiens », Mélanges Marcel Cohen. Études de linguistique, ethnographie et sciences connexes offertes par ses amis et ses élèves à l'occasion de son $80^{\circ}$ anniversaire. Avec des articles et études inédits de Marcel Cohen, réunis par David CoHEN, The Hague - Paris, Mouton (Janua Linguarum, Series Maior 27), 1970, p. 224-228.

«Variantes, variétés dialectales et contacts linguistiques en domaine arabe », Bulletin de la Société de Linguistique de Paris 68/1, 1973, p. 215-248. 
« Remarques historiques et sociolinguistiques sur les parlers arabes des Juifs maghrébins », International Journal of the Sociology of Language 30, 1981, p. 91-105. [Traduction anglaise : "Some historical and sociolinguistic observations on the Arabic dialects spoken by North African Jews ", Readings in the Sociology of Jewish Languages, ed. J. A. Fishman (Contributions to the Sociology of Jewish Languages 1), Leiden, Brill, 1985, p. 246-260].

«Les structures accentuelles de l'arabe andalou d'après Pedro de Alcalá », Comptes rendus du GLECS 24-28, 1979-1984, fasc. 2 [1986], Annexe I, $1^{\text {re }}$ partie, p. 367-382. [Avec Arlette RoTH]

«Pour un atlas linguistique et sociolinguistique de l'arabe », Actes du Premier Congrès d'études des cultures méditerranéennes d'influence arabo-berbère, éd. M. Galley, Alger, SNED, 1973, p. 63-69.

«Un questionnaire différentiel pour la dialectologie arabe », Oriente Moderno, 2000, 1 (Studi di dialettologia araba, éd. L. Bettini), p. 1-23. [Avec Dominique CAUBET et Arlette RoTH]

«Du chamito-sémitique aux dialectes arabes. Les systèmes vocaliques et les contraintes syllabiques », Dynamiques langagières en Arabophonies : variations, contacts, migrations et créations artistiques. Hommage offert à Dominique Caubet par ses élèves et collègues, éds. A. Barontini, C. Pereira, Á. Vicente \& K. Ziamari, Universidad de Zaragoza, Zaragoza \& INALCO, Paris (Estudios de dialectología Árabe 7), 2012, p. 27-55.

«À propos de racines ", Dictionnaire des racines sémitiques..., fasc. 6: W - WLHP, Leuven, Peeters, 1996, p. I-XV.

L'homme Ses bizarres idées de bonheur..., Saint-Victor-de-Morestel (Isère), Les Provinciales (diffusion Éditions du Cerf), 2008, 605 p.

Salvatore QUASIMODO, Poèmes. Choix et traduction de l'italien par David COHEN, Irène LENTIN et Stefano MANGANO, préface de Salvatore Silvano Nigro, Institut Culturel Italien de Paris (Collection Cahiers de l'Hôtel de Galliffet, nouvelle série), 2012, 252 p.

N.B. Les cinq articles signalés par un astérisque ont été repris (le premier augmenté d'un Post-scriptum) dans l'ouvrage Études de linguistique sémitique et arabe, The Hague - Paris, Mouton, 1970, 178 p. (Janua Linguarum, Series Practica 81).

On trouvera la « Bibliographie des travaux de David Cohen », établie par Geneviève Joly (avec J. Lentin et A. Lonnet) aux p. XXIII-LIII des Mélanges David Cohen. Études sur le langage, les langues, les dialectes, les littératures, offertes par ses élèves, ses collègues, ses amis, présentées à l'occasion de son quatre-vingtième anniversaire, textes réunis et édités par Jérôme LENTIN et Antoine LONNET avec l'aide d'Aziza Boucherit, Arlette Roth, Catherine Taine-Cheikh et Amor Bencheikh, Paris, Maisonneuve \& Larose, 2003, LIII+764 pages. Pour les travaux postérieurs à 2003, une bibliographie complémentaire sera publiée. 